\title{
Segmentation of Salivary Glands in Nuclear medicine Images Using Edge Detection Tools
}

\author{
Yousif Mohamed Y. Abdallah \\ Department of Radiological Sciences and medical Imaging, College of Medical Applied Sciences, \\ Majmaah University \\ y.yousif@mu.edu.sa
}

\begin{abstract}
Recognition of the Salivary glands in nuclear medicine examination is very difficult because of unclear borders and existence of noise, which affects the spatial resolution and reduces the diagnostic values of those images Therefore, image-processing programs such as MatLab, has powerful tools, which can use for solving those problems. The Morphology tool frequently applied to this problem. In this paper, I used entropyfilt function to create a texture image. This function returns an array where each output pixel contains the entropy value of the 9-by-9 neighborhood around the corresponding pixel in the salivary glands scintigraphy images. Threshold the rescaled image to segment the textures. A threshold value of 0.8 selected because it was roughly the intensity value of pixels along the boundary between the textures. The segmented images compare the binary image rough Mask to the original image. The quantitative results calculated using a measure of percentage match between ground truth and segmentation results. The percentage match (PM) measure was $99.33(p<0.05)$ and Corresponding Ratio (CR) was $-0.007 p<0.05)$. The proposed method is able to recognize the salivary glands accurately.
\end{abstract}

Keywords; Salivary glands; MatLab; Scintigraphy; Morphology.

\section{Introduction}

Image segmentation has been a long-standing problem in computer vision. It is a very difficult problem for general images, which may contain effects such as highlights, shadows, transparency, and object occlusion. Segmentation in the domain of medical imaging has some characteristics that make the segmentation task easier and difficult at the same time [1]. On the one hand, the imaging is narrowly focused on an anatomic region. The imaging context is also well defined. While context may be present to some extent in segmenting general images (e.g., indoor vs. outdoor, city vs. nature, people vs. animals), it is much more precise in a medical imaging task, where the imaging modality, imaging conditions, and the organ identity is known [2]. In addition, the pose variations are limited, and there is usually prior knowledge of the number of tissues and the Region of Interest (ROI) [3] [4]. On the other hand, the images produced in this field are one of the most challenging due to the poor quality of imaging making the anatomical region segmentation from the background very difficult. Often the intensity variations alone are not sufficient to distinguish the foreground from the background, and 
additional cues are required to isolate ROIs [5]. Finally, segmentation is often a means to an end in medical imaging. It could be part of a detection process such as tissue detection, or for the purpose of quantification of measures important for diagnosis, such as for example, lesion burden which is the number of pixels/voxels within the lesion regions in the brain [6]. In general, the information contained in an image modeled in several ways. A simple approach is to record the intensity distribution within an image via a One-dimensional (1D) histogram and use simple thresholding to obtain the various segments. Several variations on classical histogram thresholding proposed for medical image segmentation that incorporate extended image representation schemes as well as advanced information modeling [7]. Multi-dimensional histograms formed from the intensity values produced by each of the imaging protocols. It is often the case that several acquisitions are available for the same image. Spatial information: Since intensity histograms do not preserve spatial contiguity of pixels, one variation is to add spatial position $(x, y)$ or $(x, y, z)$ to form a multi-dimensional feature vector incorporating spatial layout. If the medical images are in a time sequence (e.g. moving medical imagery), then time can be added as an additional feature in the representation space [8] [9]. Thus, these approaches represent each image pixel as a feature vector in a defined multi-dimensional feature space. The segmentation task can be seen as a combination of two main processes; modeling which is the generation of a representation over a selected feature space [11]. This can be termed the modeling stage. The model components viewed as groups, or clusters in the high-dimensional space [12]. In order to be directly relevant for a segmentation task, the clusters in the model should represent homogeneous regions of the image. In general, the better the image modeling, the better the segmentation produced. Since the number of clusters in the feature space are often unknown, segmentation regarded as an unsupervised clustering task in the high dimensional feature space [13].

\subsection{Materials and Methods}

This was experimental study conducted to recognize the salivary glands scintigraphy images using image-processing program using basic morphology tools. For salivary glands scintography images treated by using image processing program (MatLab), where the recognition was studied. The scanned image saved in a PNG file format to preserve the quality of the image. Salivary glands could easily detect in nuclear medicine images if the object had sufficient contrast from the background. The Steps of recognition were as shown in figure 1. 


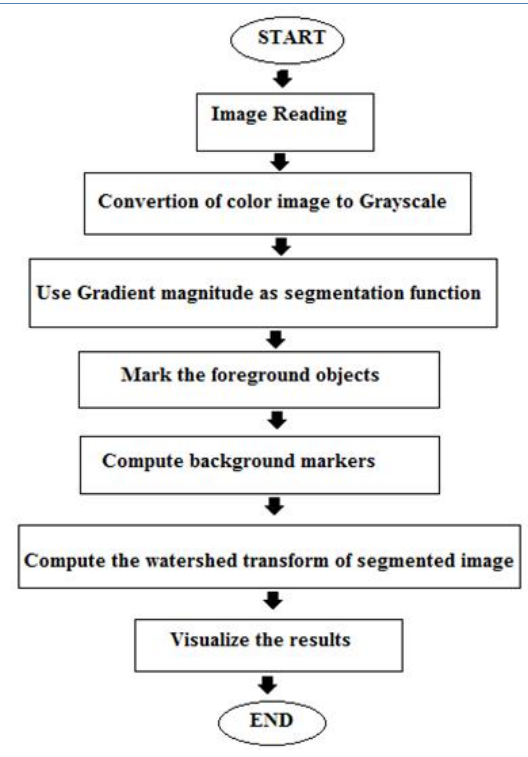

Figure 1. Steps of liver segmentation using MatLab program

\section{The results}

This was experimental study conducted to recognize the salivary glands scintigraphy images using image-processing program using basic morphology tools. The segmentation of image started firstly by reading image as shown in (Figure 1 ).

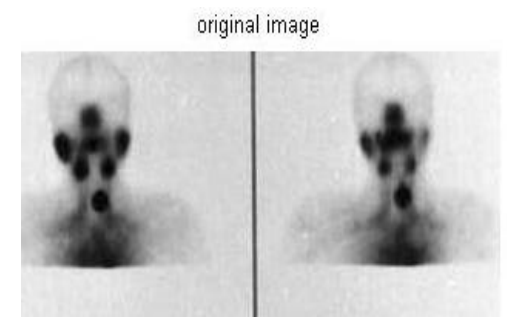

Figure 2. Original image used for Segmentation

The salivary glands are present in this image and they can be seen in them entirety. I detected or segmented those glands. The object segmented differs greatly in contrast from the background image. Operators that calculate the gradient of an image in contrast could detect changes. The gradient image could calculate and a threshold could apply to create a binary mask containing the segmented cell. First, I use edge and the Sobel operator to calculate the threshold value. I then tune the threshold value and use edge again to obtain a binary mask that contains the segmented salivary glands as shown in (Figure 2).

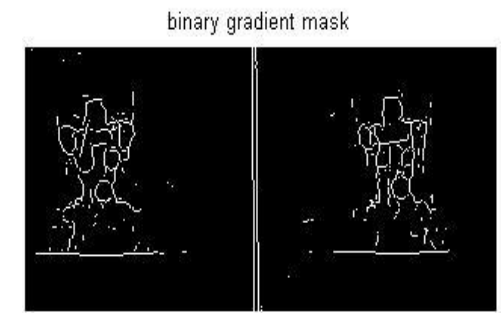

Figure 2. Binary Gradient Magnitude Mask 
The binary gradient mask shows lines of high contrast in the image. These lines do not quite delineate the outline of the object of interest. Compared to the original image, I can see gaps in the lines surrounding the object in the gradient mask. These linear gaps will disappear if the Sobel image is dilated using linear structuring elements, which we can create with the strel function. The binary gradient mask dilated using the vertical structuring element followed by the horizontal structuring element. The imdilate function dilates the image as shown in figure 3.

dilated gradient mask
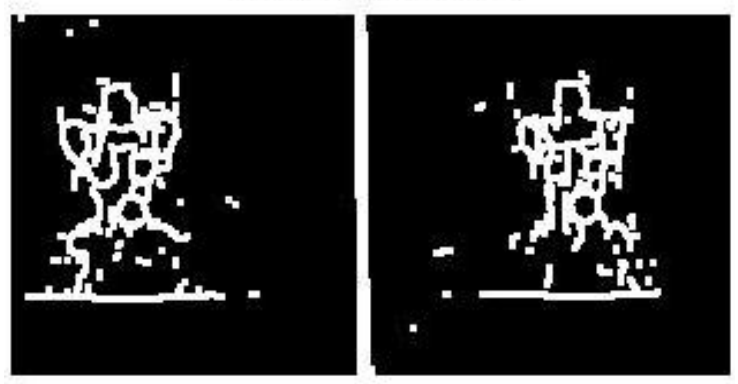

Figure 3. The dilated gradient mask

The dilated gradient mask shows the outline of the thyroid quite nicely, but there are still holes in the interior of the lungs. To fill these holes we use the imfill function as shown in figure 4.

binary image with filled holes
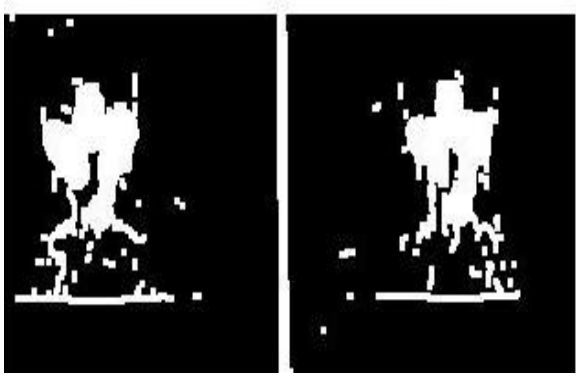

Figure 4. Binary image with filled holes

The salivary gland have been successfully segmented, but it is not the only object that has been found. Any objects that connected to the border of the image can be removed using the imclearborder function. The connectivity in the imclearborder function was set to 4 to remove diagonal connections as shown in figure 5 .

cleared border image

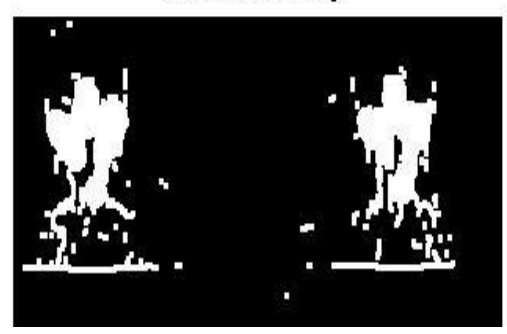

Figure 5. Cleared Border image 
Yousif Mohamed Y. Abdallah; Segmentation of Salivary Glands in Nuclear medicine Images Using Edge Detection Tools. Journal of Biomedical Engineering and Medical Imaging, Volume 3, No 2, April (2016), pp 1-6

Finally, in order to make the segmented object look natural, we smoothen the object by eroding the image twice with a diamond structuring element. I create the diamond structuring element using the strel function as shown in figure 6.

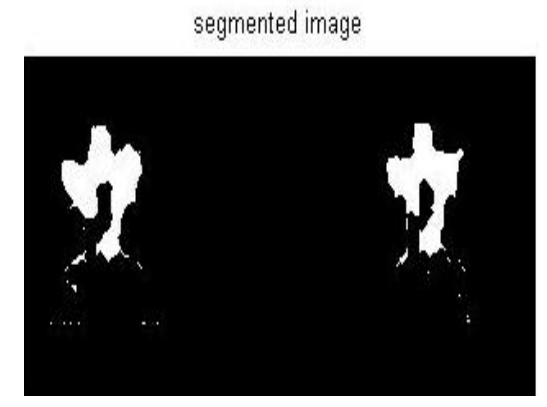

Figure 6. The segmented image

An alternate method for displaying the segmented object would be to place an outline around the segmented thyroid. The outline created by the bwperim function figure 7 .

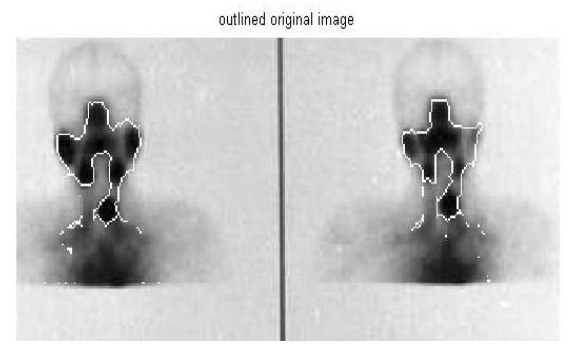

Figure 7. The Outlined original image

\section{Conclusion}

The smoothing of the object by eroding the image twice with a diamond-structuring element using the strel function will create the diamond-structuring element, which is useful of natural look of segmented object. So conclusion of this paper that edge detection and basic morphology tools are best tools to detect salivary glands. The detection of the noise is a complex procedure, which is difficult to detect by naked eye so that image analysis should perform by using powerful image processing. A watershed transform Algorithm lungs segmentation method proposed in this study. Proposed method is able to determine the salivary glands boundaries accurately. It is able to segment salivary glands and improves radiological analysis and diagnosis.

\section{REFERENCES}

[1] Abdallah, Y., Hayder, A., and Wagiallah ,E., Automatic Enhancement of Mammogram using Contrast Algorithm. International Journal of Science and Research (IJSR), 2014. 3(9): p. 1886-1891.

[2] Abdelwahab, R., and Abdallah, Y., Hayder, A., and Wagiallah ,E., Application of Texture Analysis Algorithm for Data Extraction in Dental X-Ray Images. International Journal of Science and Research (IJSR), 2014. 3(10): p. 1934-1939. 
[3] Abdallah, Y., Alkhir, M., Algaddal, A., Improvement of Brain Tumors Detection Using Markers and Boundaries Transform. International Journal of Science and Research (IJSR), 2015. 4(1): p. 2372-2378.

[4] Bidgood, D. \& Horii, S., Introduction to the ACR-NEMA DICOM standard. RadioGraphics, 1992. 12: p. 345355.

[5] Lehmann, T.M.; Gönner, C. \& Spitzer, K., Survey: Interpolation Methods in Medical Image Processing. IEEE Transactions on Medical Imagin: 1999. 18(11): p. 1049-1075

[6] Li, G. \& Miller, R.W., Volumetric Image Registration of Multi-modality Images of CT, MRI and PET, Biomedical Imaging. Youxin Mao (Ed.), 2010.

[7] Abdallah, Y., Application of Analysis Approach in Noise Estimation, Using Image Processing Program. Lambert Publishing Press GmbH \& Co. KG, Germany, 2011.

[8] Lyra, M.; Sotiropoulos, M., Lagopati, N. \& Gavrilleli, M. , Quantification of Myocardial Perfusion in 3D SPECT images - Stress/Rest volume differences, Imaging Systems and Techniques (IST), IEEE International Conference 2010: p. $31-35$

[9] Narasimha-lyer, H., et al., Automatic Identification of Retinal Arteries and Veins From Dual-Wavelength Images Using Structural and Functional Features. Biomedical Engineering, IEEE Transactions, 2007. 54(8): p. 1427-1435.

[10] Abdallah, Y., and Wagiallah, E., Segmentation of Thyroid Scintography Using Edge Detection and Morphology Filters. International Journal of Science and Research (IJSR), 2014. 3(11): p. 2768-2772.

[11] Abdallah, Y., and Hassan, A., Segmentation of Brain in MRI Images Using Watershed-based Technique. International Journal of Science and Research (IJSR), 2015. 4(1): p. 683-688.

[12] Hong, S., et al., Optimal scheduling of tracing computations for real-time vascular landmark extraction from retinal fundus images. Information Technology in Biomedicine, IEEE Transactions on, 2001. 5(1): p. 77-91.

[13] Pinz, A., et al., Mapping the human retina. Medical Imaging, IEEE Transactions on, 1998. 17(4): p. 606-619.

[14] Abdallah, Y., and Yousef R., Augmentation of X-Rays Images using Pixel Intensity Values Adjustments. International Journal of Science and Research (IJSR), 2015. 4(2): p. 2425-2430.

[15] Meyer, Fernand, "Topographic distance and watershed lines," Signal Processing , Vol. 38, July 1994, pp. 113-125 